\title{
AVALIAÇÃO DE ERROS EM EXAMES RADIOGRÁFICOS INTRABUCAIS REALIZADOS POR ACADÊMICOS DE ODONTOLOGIA
}

\section{EVALUATION OF ERRORS IN INTRAORAL RADIOGRAPHS STUDENTS OF DENTISTRY}

\author{
Deborah Montenegro Mendonça* \\ Max Eduardo Barroso de Amorim** \\ Pollyanna de Oliveira Medina"** \\ Ary de Oliveira Alves Filho**** \\ Tânia Cristina Chicre Alcântara de Brito"*n* \\ Nikeila Chacon de Oliveira Conde ${ }^{\cdots \cdots \cdots}$
}

\begin{abstract}
RESUMO
O objetivo deste estudo foi analisar as radiografias intrabucais realizadas no Setor de Radiologia, determinando o tipo de erro mais comum e relacionando-o com o tempo e região radiografada. Para tal, dois examinadores previamente calibrados entre si $(K a p p a=0,83)$ observaram as radiografias quanto ao erro de técnica e erro de processamento. Os dados foram analisados pela estatística descritiva e através do teste do qui-quadrado de Pearson e Fisher, quando aplicável. Os resultados demonstraram que, do total de 281 radiografias analisadas, em $224(79,7 \%)$ foi identificado algum tipo de erro. Quanto aos erros de técnica, a maior porcentagem ocorreu por angulagem vertical incorreta em $79(28,1 \%)$, e quanto aos erros de processamento, $42(14,9 \%)$ por manchas. Quando avaliada a relação do tipo de erro com a arcada radiografada, a angulagem vertical manteve-se como o erro mais comum na arcada superior 58 (41,1\%), porém, na arcada inferior o erro mais comum foi por corte do dente ou parte dele, 39 (35,5\%). Quando avaliado o tempo da disciplina e o tipo de erro, observou-se diferença estatisticamente significante entre o início e o término da Disciplina quanto à presença de erro de angulagem vertical $(p=0,001)$. Pode-se concluir que o erro de técnica mais comum foi angulagem vertical e de processamento (manchadas), havendo diminuição durante a prática do ensino.
\end{abstract}

Descritores: Fotografia dentária • Radiografia dentária • Controle de qualidade

\section{ABSTRACT}

The aim of this study was to analyze the intraoral radiographs performed in the Radiology, determining the most common type of error and relating it to the time and region imaged. To this end, two calibrated examiners together $($ Kappa $=0.83$ ) observed radiographs as technical and processing errors. The data were analyzed using descriptive statistics and chi-square test and Fisher, when applicable. The results showed that from a total of 281 analyzed radiographs, in $224(79.7 \%)$ were identified some types of error. The errors of technique, the highest percentage was incorrect vertical angulation by $79(28.1 \%)$, and processing errors, $42(14.9 \%)$ of spots. When evaluating the relationship of the type of error with the arcade imaged, the vertical angulation remained as the most common mistake in the upper arch, 58 (41.1\%); however, in the lower arch was the most common mistake by cutting the tooth / 39 part of it (35.5\%). When measured time of discipline and type of error, there was a statistically significant difference between the beginning and the end of the Discipline for the presence of vertical angulation error $(p=0.001)$. It can be concluded that the most common technique error was the vertical angulation and processing (stained), with decrease during teaching practice.

Descriptors: Photography, dental • Radiography, dental • Quality control

* Acadêmica da Faculdade de Odontologia-Universidade Federal do Amazonas, Manaus-AM, Brasil. Email: deborahmmendonca@gmail.com

** Professor Assistente, Especialista em Cirurgia e Traumatologia em Buco-Maxilo-Facial da Faculdade de Odontologia-Universidade Federal do Amazonas, Manaus-AM, Brasil. E-mail: maxamorim@bol.com.br

*** Professora Auxiliar, Especialista em Odontopediatria da Faculdade de Odontologia-Universidade Federal do Amazonas, Manaus-AM, Brasil. E-mail: polly.medina@hotmail.com

**** Professor Assistente, Mestre em Odontopediatria da Faculdade de Odontologia-Universidade Federal do Amazonas, Manaus-AM, Brasil. E-mail: draryfilho@globo.com

****** Professora Adjunto, Doutora em Odontologia da Faculdade de Odontologia-Universidade Federal do Amazonas, Manaus-AM, Brasil. E-mail: taniachicre@hotmail.com

****** Professora Adjunto, Doutora em Estomatologia, Diretora da Faculdade de Odontologia-Universidade Federal do Amazonas, Manaus-AM, Brasil. E-mail: nikeilaconde@gmail.com 


\section{N T R O D UÇÃO}

O uso dos raios $X$ constitui ferramenta essencial na clínica odontológica como avaliação complementar ao exame clínico odontológico ${ }^{1}$.

De acordo com a Organização Mundial de Saúde (OMS), a baixa qualidade das imagens radiográficas pode ser um fator responsável pela redução na precisão dos diagnósticos ${ }^{2}$.

A exposição à radiação pode provocar efeitos deletérios ao organismo, independentemente da quantidade de exposição. Uma pequena quantidade de radiação não será suficiente para provocar uma manifestação clínica ou genética, mas certamente poderá provocar uma reação celular com quebra e desorganização de moléculas. Não se conhecem, com precisão, os efeitos biológicos da radiação para pequenas doses tanto para desenvolver uma lesão (nível somático), como para provocar mutação (nível genético). Em nível somático, ocorre destruição de tecidos em que a radiossensibilidade é maior, como em células de tecidos vasculares e as pertencentes aos órgãos sexual e oftálmico. Em nível genético, as mutações ocorrem por quebras de cromossomos que contêm os gens, ocorrendo reorganização aleatória e alterando o padrão hereditário ${ }^{3}$.

Em junho de 1998, o Ministério da Saúde publicou a Portaria $n^{\circ} 453$ regulamentando a utilização dos raios X para diagnóstico em Medicina e Odontologia 4 . Foi determinada a implantação dos Programas de Controle de Qualidade em radiologia odontológica, que consiste na monitoração sistemática e controlada de equipamentos e procedimentos, com o objetivo de apontar falhas e defeitos que possam comprometer a qualidade das imagens radiográficas, dos equipamentos e de todos os procedimentos relacionados a esse processo, evitando-se, assim, diagnósticos equivocados ou inconclusivos, que acarretariam doses desnecessárias da repetição dos exames. De acordo com o item 3.27 dessa Portaria, compete ao Responsável Técnico responsabilizar-se pelos procedimentos radiológicos a que são submetidos os pacientes, levando-se em conta os princípios e requisitos de prote- ção radiológica estabelecidos neste Regulamento, devendo: a) Assegurar que nos procedimentos radiológicos sejam utilizados as técnicas e os equipamentos adequados e b) Zelar para que as exposições de pacientes sejam as mínimas necessárias para atingir o objetivo do procedimento radiológico requisitado, levando em conta os padrões aceitáveis de qualidade de imagem e as restrições conferidas pelos níveis de referência de radiodiagnóstico estabelecidos neste Regulamento ${ }^{4}$.

Os exames radiográficos são rotineiramente empregados como auxiliares da avaliação clínica dos pacientes, pois podem fornecer informações complementares necessárias à elaboração do diagnóstico, planejamento, realização e proservação do tratamento ${ }^{5}$. Falhas na execução das técnicas ou durante o processamento radiográfico dos filmes resultam em imagens radiográficas insatisfatórias, que podem gerar interpretações errôneas, além de acarretar repetição do exame, com consequente aumento da exposição dos pacientes à radiação ${ }^{5,6}$.

Com base nesses princípios e visando à utilização do exame radiográfico de maneira racional, surge a necessidade do domínio da técnica radiográfica ainda na vida acadêmica, para permitir a execução de um diagnóstico e tratamento adequados para o paciente. Dessa forma, os objetivos deste estudo foram analisar as radiografias intrabucais realizadas no Setor de Radiologia durante a disciplina de Radiologia, determinar o principal tipo de erro radiográfico presente, comparar erros mais comuns nos procedimentos, no início e término da Disciplina, e estabelecer relação entre número de erros radiográficos e arcada radiografada.

\section{MATERIAIS E MÉTODOS}

Foram analisadas radiografias intrabucais (periapicais, interproximais e oclusais) realizadas durante a Disciplina de Radiologia da Faculdade de Odontologia da Universidade Federal do Amazonas, no período de agosto a novembro de 2011. As radiografias avaliadas na pesquisa foram aquelas apresentadas ao docente responsável como resultado final do exame.

O projeto foi encaminhado ao Comitê
MENDONÇA DM AMORIM MEB MEDINA PO ALVES FILHO AO BRITO TCCA

CONDE NCO

AVALIAÇÃO DE ERROS EM EXA MES RADIOGRÁFICOS INTRABUCAIS REALIZADO POR ACADEMICOS DE ODONTOLOGIA / UFAM

209
REV, ODONTOL.

UNIV. CID. São PAULO

$2013 ; 25(3): 208-$ 15，SET-DEZ 
MENDONÇA DM AMORIM MEB

MEDINA PO

ALVESFILHO AO

BRITO TCCA

CONDE NCO

AVALIAÇÃO DE ERROS EM EXAMES RADIOGRÁFICOS INTRABUCAIS

REALIZADO POR

ACADEMICOS DE ODONTOLOGIA/ UFAM

\section{0}

REV, ODONTOL.

UNIV, CID, SÃO

PAULO

$2013 ; 25(3): 208-$

15, SET-DEZ de Ética e Pesquisa (CEP) da Universidade Federal do Amazonas para análise sob o CAAE-0099.0.115.000-11 sendo considerada dispensável sua apresentação ao CEP, uma vez que não envolveu dados de seres humanos direta ou indiretamente.

Foram incluídas no estudo todas as radiografias intrabucais realizadas pelos métodos periapical da bissetriz, paralelismo, interproximal, oclusal e as não identificadas, consideradas insatisfatórias (por qualquer tipo de erro), durante a Disciplina de Radiologia, e excluídas as radiografias intrabucais realizadas em crianças e em pacientes especiais, pela maior probabilidade de erros de técnica e pela não colaboração dos pacientes.

Para análise das radiografias insatisfatórias foram adotados os seguintes critérios avaliativos de referência para o estudo ${ }^{6}$ :

Erros de técnica radiográfica: a) o enquadramento: foi subdividido em posicionamento inadequado do filme, incluindo filmes com longo eixo contrário, cortes de um dente ou parte dele na região radiografada e aparecimento de meia lua; b) a angulagem: foi subdividida em angulagem vertical (alongamento e encurtamento) e angulagem horizontal; c) tempo de exposição: radiografias com subexposição (claras) ou superexposição (escuras), que não permitiram a interpretação das imagens radiográficas; d) borradas ou tremidas: radiografias com contornos sem nitidez, devido ao deslocamento do filme, aparelho ou paciente durante a exposição; e) filme exposto pelo lado da lamínula de chumbo: radiografias executadas com a película invertida em relação à sua face sensível;

Erros de processamento radiográfico: Radiografias manchadas, amareladas, riscadas, reveladas parcialmente ou com impressões digitais.

A análise das radiografias foi realizada por dois examinadores, a acadêmica e um profissional, previamente calibrados entre si (Teste Kappa =0,83) com auxílio de, negatoscópio, lupa e sala escura.

Os dados foram apresentados por meio de tabelas, onde foram calculadas as frequências absolutas simples e relativa. $\mathrm{Na}$ análise das variáveis categóricas utilizou-se a estatística de teste do qui-quadrado de Pearson, sendo que na impossibilidade de aplicação do teste de Pearson, para tabelas $2 \times 2$, utilizou-se o teste exato de $\mathrm{Fi}$ sher. O software utilizado na análise foi o programa Epi-Info versão 7 para Windows, que é desenvolvido e distribuído gratuitamente pelo CDC (www.cdc.gov/ epiinfo). O nível de significância fixado nos testes foi de $5 \%$.

\section{RESULTADOS}

No total, foram analisadas 281 radiografias, dentre elas $224(79,7 \%)$ insatisfatórias. Dentre as técnicas utilizadas, 245 $(87,2 \%)$ foram radiografias periapicais, 22 $(7,8 \%)$ interproximais, $8(2,8 \%)$ oclusais e $6(2,2 \%)$ não identificadas (Tabela 1$)$.

$\mathrm{Na}$ análise da condição da radiografia, foi constatada diferença estatística ao nível de $5 \%$ em relação ao momento da radiografia $(p<0,001)$, onde se observou que no início da disciplina o percentual de radiografias insatisfatórias era de 149 $(88,2 \%)$, passando para $75(67 \%)$ no final da disciplina (Tabela 2).

Quanto ao erro de técnica, o maior erro encontrado na maioria das radiografias foi na angulagem vertical, totalizando $79(28,1 \%)$ erros, seguido de 73 (26\%) erros no enquadramento (corte do dente ou parte dele) e $51(18,1 \%)$ erros de meia lua. Quanto ao erro de processamento, o mais cometido foi de radiografias manchadas totalizando $42(14,9 \%)$ erros, seguido de $21(7,5 \%)$ erros de radiografias riscadas, e $11(3,9 \%)$ erros com impressão digital. (Tabela 3).

Também foi constatada diferença estatística do momento da radiografia em relação aos erros de angulagem vertical $(p$ $<0,001)$, claras $(p=0,048)$, cortes de um dente ou parte dele $(p<0,001)$, sendo que neste último caso o percentual aumentou no fim da disciplina (Tabela 4).

$\mathrm{Na}$ Tabela 5 pode-se observar que o maior número de erros de técnica radiográfica encontrados foi na arcada superior em angulagem vertical com $58(41,1 \%)$. $\mathrm{Na}$ arcada inferior o maior erro de técnica encontrado foi em corte do dente ou parte dele com 39 (35,5\%) de erros, enquanto na arcada superior/inferior os maiores erros encontrados foram de angulagem vertical e horizontal, igualmente de 5 (25\%) para cada. Em relação aos erros de proces- 
Tabela 1. Distribuição segundo a frequência do momento da radiografia, região, técnica utilizada e condição das radiografias realizadas na disciplina de radiologia da UFAM, Manaus - AM

\begin{tabular}{lll}
\hline \hline Variáveis $(\mathrm{n}=281)$ & $\mathrm{f}_{\mathrm{i}}$ & $\%$ \\
\hline $\begin{array}{l}\text { Momento da radiografia } \\
\text { Início da disciplina }\end{array}$ & 169 & 60,1 \\
Fim da disciplina & 112 & 39,9 \\
Região da radiografia & & \\
$\quad$ Superior & 141 & 50,2 \\
Inferior & 110 & 39,1 \\
Superior e inferior & 20 & 7,1 \\
Não identificada & 10 & 3,6 \\
Técnica utilizada & & \\
Periapical & 245 & 87,2 \\
Interproximal & 22 & 7,8 \\
Oclusal & 8 & 2,8 \\
Não identificada & 6 & 2,2 \\
Condição radiográfica & & \\
Insatisfatória & 224 & 79,7 \\
\hline \hline
\end{tabular}

$\mathrm{f}_{\mathrm{i}}=$ frequência absoluta simples.

Tabela 2. Distribuição segundo a frequência da condição em relação ao momento, região e técnica utilizada nas radiografias realizadas no setor de radiologia da UFAM, Manaus - AM.

\begin{tabular}{lcccc}
\hline \hline & \multicolumn{4}{c}{ Condição da radiografia } \\
Variáveis & \multicolumn{3}{c}{ Insatisfatória } \\
& $\mathrm{f}_{\mathrm{i}}$ & $\%$ & Total & $\mathrm{p}^{*}$ \\
\hline Momento da radiografia & & & & $<\mathbf{0 , 0 1}$ \\
Início da disciplina & 149 & 88,2 & 169 & \\
Fim da disciplina & 75 & 67,0 & 112 & \\
Região da radiografia & & & & 0,141 \\
Superior & 115 & 81,6 & 141 & \\
Inferior & 81 & 73,6 & 110 & \\
Superior e inferior & 18 & 90,0 & 20 & $*$ \\
Técnica utilizada & & & & \\
Periapical & 190 & 77,6 & 245 & \\
Interproximal & 20 & 90,9 & 22 & \\
Oclusal & 8 & 100,0 & 8 & \\
\hline \hline
\end{tabular}

$\mathrm{f}_{\mathrm{i}}=$ frequência absoluta simples; ${ }^{*}$ Teste do qui-quadrado de Pearson; ${ }^{*}$ Não é possível aplicar a estatística de teste devido às restrições do qui-quadrado (VIEIRA, 2004).

Valor de " $\mathrm{p}$ " em negrito itálico indica diferença estatisticamente significante ao nível de 5\%.

Tabela 3. Distribuição segundo a frequência das radiografias insatisfatórias realizadas no setor de radiologia da UFAM, Manaus - AM.

\begin{tabular}{lll}
\hline \hline Variáveis $(n=281)$ & $\mathrm{f}_{\mathrm{i}}$ & $\%$ \\
\hline
\end{tabular}

\section{Erros de técnica radiográ-} fica

Angulagem vertical

Cortes de um dente ou parte dele

7326,0

Incidência do feixe central (Meia lua)

$51 \quad 18,1$

Claras

Angulagem horizontal $\quad 36 \quad 12,8$

Deslocamento do filme

Escuras

$21 \quad 7,5$

Filme com longo eixo

contrário

$5 \quad 1,8$

\section{Erros de processamento}

Manchadas

$42 \quad 14,9$

Riscadas

$21-7,5$

Impressão digital

113,9

Amareladas

$9 \quad 3,2$

Reveladas parcialmente

$\mathrm{f}_{\mathrm{i}}=$ frequência absoluta simples

\author{
MENDONÇA DM \\ AMORIM MEB \\ MEDINA PO \\ ALVES FILHO AO \\ BRITO TCCA \\ CONDE NCO \\ AVALIAÇÃO DE \\ ERROS EM EXA- \\ MES RADIOGRÁFI- \\ COS INTRABUCAIS \\ REALIZADO POR \\ ACADEMICOS DE \\ ODONTOLOGIA/ UFAM
}




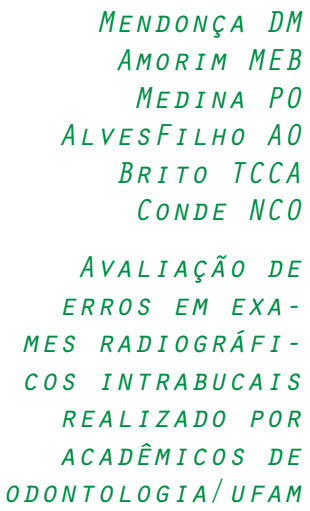

212

REV, ODONTOL

UNIV. CID. SÃO

PAULO :

$2013 ; 25(3): 208-$

15, SET-DEZ

Tabela 4. Distribuição segundo a frequência do momento da disciplina realizada no setor de radiologia da UFAM, Manaus - AM.

\begin{tabular}{|c|c|c|c|c|c|c|}
\hline \multicolumn{7}{|c|}{ Momento da disciplina } \\
\hline \multirow[t]{2}{*}{ Variáveis } & \multicolumn{2}{|c|}{$\begin{array}{l}\text { Início } \\
(\mathrm{n}=169)\end{array}$} & \multicolumn{2}{|c|}{$\begin{array}{l}\text { Fim } \\
(\mathrm{n}=112)\end{array}$} & \multirow[b]{2}{*}{ Total } & \multirow[b]{2}{*}{$\mathrm{p}^{*}$} \\
\hline & $f_{i}$ & $\%$ & $f_{i}$ & $\%$ & & \\
\hline \multicolumn{7}{|l|}{ Erros de técnica radiográfica } \\
\hline Angulagem vertical & 61 & 36,1 & 18 & 16,1 & 79 & $<0,001$ \\
\hline Cortes de um dente ou parte dele & 28 & 16,6 & 45 & 40,2 & 73 & $<0,001$ \\
\hline Meia lua & 36 & 21,3 & 15 & 13,4 & 51 & 0,092 \\
\hline Claras & 33 & 19,5 & 12 & 10,7 & 45 & 0,048 \\
\hline Angulagem horizontal & 20 & 11,8 & 16 & 14,3 & 36 & 0,547 \\
\hline Deslocamento do filme & 11 & 6,5 & 10 & 8,9 & 21 & 0,450 \\
\hline Escuras & 4 & 2,4 & 2 & 1,8 & 6 & $0,547^{* *}$ \\
\hline Filme com longo eixo contrário & 4 & 2,4 & 1 & 0,9 & 5 & $0,338^{* *}$ \\
\hline \multicolumn{7}{|l|}{ Erros de processamento } \\
\hline Manchadas & 29 & 17,2 & 13 & 11,6 & 42 & 0,201 \\
\hline Riscadas & 12 & 7,1 & 9 & 8,0 & 21 & 0,770 \\
\hline Impressão digital & 9 & 5,3 & 2 & 1,8 & 11 & $0,116^{* *}$ \\
\hline Amareladas & 4 & 2,4 & 5 & 4,5 & 9 & $0,260 * *$ \\
\hline Reveladas parcialmente & 8 & 4,7 & 1 & 0,9 & 9 & $0,069 * *$ \\
\hline
\end{tabular}

$\mathrm{fi}$ = frequência absoluta simples; * Teste do qui-quadrado de Pearson; ** Teste exato de Fisher. Valor de " $\mathrm{p}$ " em negrito itálico indica diferença estatisticamente significante ao nível de $5 \%$.

$\mathrm{f}_{\mathrm{i}}=$ frequência absoluta simples; ${ }^{*}$ Teste do qui-quadrado de Pearson; $* *$ Teste exato de Fisher.

Valor de "p" em negrito itálico indica diferença estatisticamente significante ao nível de 5\%.

samento, a arcada superior/inferior apresentou $4(20 \%)$ com erros por impressão digital, seguidos de $23(16,3 \%)$ na arcada superior e $16(14,5 \%)$ na arcada inferior por radiografias manchadas.

\section{I SCUSSÃO}

A imagem radiográfica de qualidade deve apresentar os requisitos de máxima nitidez ou definição das estruturas anatômicas, mínima distorção, enquadramento correto do filme, ausência de artefatos ${ }^{5}$, além de densidade e contraste adequa$\mathrm{dos}^{7}$.

De acordo com Matheus et al. ${ }^{8}$ (2000), a presença de falhas em radiografias resulta em imagens inadequadas, que podem gerar interpretações errôneas.

Observa-se que a maioria das radiografias foram executadas (Tabela 1) pela técnica radiográfica periapical $245(87,2 \%)$ em comparação às técnicas oclusal $8(2,8 \%)$ e interproximal $22(7,8 \%)$, demonstrando que o exame radiográfico periapical foi solicitado com maior frequência para fins de diagnóstico. A radiografia periapical é um exame complementar capaz de fornecer informações que não podem ser evidenciadas clinicamente, sendo muito utilizada pelos cirurgiões-dentistas durante o diagnóstico, planejamento, execução e proservação de um caso clínico no tratamento odontológico ${ }^{9}$.

Em estudo longitudinal realizado por Carvalho et al. ${ }^{6}$ (2009) foi observada uma redução do número de erros radiográficos ao longo da prática acadêmica. Tais resultados estão de acordo com os dados constatados neste estudo (Tabela 2) onde houve redução significativa de radiografias insatisfatórias de 149 (88,2\%) para 75 $(67 \%)(p \leq 0,001)$. A diminuição da porcentagem de erros é considerada previsível, uma vez que, após o cumprimento de $50 \%$ da carga horária da disciplina, o acadêmico já tem realizado o número de repetições suficientes para fixar a execução da técnica radiográfica. 
Tabela 5. Distribuição segundo a frequência da arcada em relação à condição das radiografias realizadas no setor de radiologia da UFAM, Manaus - AM.

\begin{tabular}{|c|c|c|c|c|c|c|c|c|}
\hline \multicolumn{9}{|c|}{ Arcada radiografada } \\
\hline \multirow[t]{2}{*}{ Variáveis } & \multicolumn{2}{|c|}{$\begin{array}{l}\text { Superior } \\
(\mathrm{n}=141)\end{array}$} & \multicolumn{2}{|c|}{$\begin{array}{l}\text { Inferior } \\
(\mathrm{n}=110)\end{array}$} & \multicolumn{2}{|c|}{$\begin{array}{l}\text { Superior/ } \\
\text { Inferior } \\
(\mathrm{n}=20)\end{array}$} & \multirow[b]{2}{*}{ Total } & \multirow[b]{2}{*}{$\mathrm{p}^{*}$} \\
\hline & $\mathrm{f}_{\mathrm{i}}$ & $\%$ & $f_{i}$ & $\%$ & $f_{i}$ & $\%$ & & \\
\hline \multicolumn{9}{|l|}{ Erros de técnica radiográfica } \\
\hline Angulagem vertical & 58 & 41,1 & 16 & 14,5 & 5 & 25,0 & 79 & $<0,001$ \\
\hline $\begin{array}{l}\text { Cortes de um dente ou parte } \\
\text { dele }\end{array}$ & 29 & 20,6 & 39 & 35,5 & 1 & 5,0 & 69 & 0,002 \\
\hline Meia lua & 20 & 14,2 & 27 & 24,5 & 2 & 10,0 & 49 & 0,066 \\
\hline Claras & 27 & 19,1 & 17 & 15,5 & 1 & 5,0 & 45 & 0,258 \\
\hline Angulagem horizontal & 22 & 15,6 & 8 & 7,3 & 5 & 25,0 & 35 & 0,037 \\
\hline Deslocamento do filme & 8 & 5,7 & 11 & 10,0 & 1 & 5,0 & 20 & 0,392 \\
\hline Escuras & 2 & 1,4 & 2 & 1,8 & - & - & 6 & $* *$ \\
\hline $\begin{array}{l}\text { Filme com longo eixo contrá- } \\
\text { rio }\end{array}$ & 2 & 1,4 & 2 & 1,8 & 1 & 5,0 & 5 & $* *$ \\
\hline \multicolumn{9}{|l|}{ Erros de processamento } \\
\hline Manchadas & 23 & 16,3 & 16 & 14,5 & 2 & 10,0 & 41 & 0,744 \\
\hline Riscadas & 12 & 8,5 & 8 & 7,3 & 1 & 5,0 & 21 & 0,835 \\
\hline Impressão digital & 5 & 3,5 & 1 & 0,9 & 4 & 20,0 & 10 & $* *$ \\
\hline Amareladas & 6 & 4,3 & 2 & 1,8 & - & - & 9 & $* *$ \\
\hline Reveladas parcialmente & 2 & 1,4 & 3 & 2,7 & - & - & 5 & $* *$ \\
\hline
\end{tabular}

$\mathrm{f}_{\mathrm{i}}=$ frequência absoluta simples; * Teste do qui-quadrado de Pearson; ** Teste exato de Fisher. Valor de "p" em negrito itálico indica diferença estatisticamente significante ao nível de $5 \%$.

Neste trabalho, o maior erro de técnica encontrado (Tabela 3) foi o de angulagem vertical com $79(28,1 \%)$ e essa frequência foi superior aos resultados de Matheus et al. ${ }^{8}$ (2000), Kreich et al. ${ }^{5}$ (2002), dos Anjos Pontual et al. ${ }^{10}$ (2005), Felippe et al. ${ }^{11}(2009)$, Carvalho et al. ${ }^{6}$ (2009), Dias et al. ${ }^{12}$ (2009) e Guimarães et al. ${ }^{13}$ (2011). Apesar de Carvalho et al. ${ }^{6}$ (2009) afirmarem que o erro de angulagem vertical se deve muitas vezes à não utilização da tabela de ângulos verticais durante a tomada, no presente estudo, os exames radiográficos foram realizados com o auxílio da tabela de ângulos fixados no Setor de Radiologia, indicando que a porcentagem de erro pode estar relacionada à não padronização das demais orientações da técnica, não avaliadas neste estudo, como a posição da cabeça do paciente em relação ao Plano Oclusal ou posicionamento da película radiográ- fica.

Quanto ao erro de processamento, o mais cometido foi de radiografias manchadas, totalizando $42(14,9 \%)$. A alta porcentagem de radiografias manchadas pode estar relacionada ao não cumprimento do tempo recomendado de lavagem da radiografia após a fixação ${ }^{8}$, no entanto, as manchas podem estar relacionadas a outros fatores como respingos de soluções ou a colgadura com resíduo de líquido ${ }^{11} \mathrm{e}$ fixação insuficiente ${ }^{8}$.

Relacionando o tipo de erro à arcada examinada (Tabela 5), observa-se que 39 $(35,5 \%)$ dos erros na arcada inferior ocorreram pelo corte do dente ou parte dele, justificado pelo incômodo ao paciente e dificuldade de posicionamento do filme na região de assoalho bucal ${ }^{1,3,14}$. Para a arcada superior/inferior, foram observados erros na angulagem vertical e horizontal igualmente em 5 (25\%), divergindo de
MENDONÇA DM AMORIM MEB

MEDINA PO

ALVES FILHO AO

BRITO TCCA

CONDE NCO

AVALIAÇÃO DE ERROS EM EXA-

MES RADIOGRÁFI-

COS INTRABUCAIS

REALIZADO POR

ACADEMICOS DE

ODONTOLOGIA/UFAM
REV, ODONTOL.

UNIV. CID. São

PAULO

$2013 ; 25(3): 208-$

15，SET-DEZ 
MENDONÇA DM AMORIM MEB

MEDINA PO

ALVESFILHO AO

BRITO TCCA

CONDE NCO

AVALIAÇÃO DE

ERROS EM EXA-

MES RADIOGRÁFI -

COS INTRABUCAIS

REALIZADO POR

ACADEMICOS DE

ODONTOLOGIA/ UFAM

214
Guimarães et al. ${ }^{15}(2009)$ para os quais o erro prevalente foi de angulagem horizontal, resultando em sobreposição das faces proximais, inviabilizando o diagnóstico.

Outros fatores relacionados ao estudo, porém não avaliados, estão relacionados ao tempo de docência dos professores que orientaram os alunos, número de repetições executadas por cada aluno e métodos radiográficos empregados para técnica periapical.

\section{CONCLUSÕES}

Os resultados demonstraram que o erro mais comum para o critério técnica foi de angulagem vertical e, para o critério processamento, o erro mais observado foi de radiografias manchadas. A porcentagem de radiografias insatisfatórias diminuiu significativamente ao longo do período. Quando comparados arcada e tipo de erro, na arcada superior a angulagem vertical permaneceu como o erro mais comum, porém para a arcada inferior, o corte do dente ou parte dele revelou maior ocorrência.

\section{REFERÊNCIAS}

1. Whaites E. Princípios de radiologia odontológica. 3. ed. Porto Alegre: Artmed; 2003.

2. Teunen D. The European Directive on health protection of individuals against the dangers of ionising radiation in relation to medical exposures (97/43/EURATOM). J Radiol Prot 1998 Jun;18(2):133-7.

3. White S, Pharoah M. Radiologia oral: fundamentos e interpretação. 5. ed. Rio de Janeiro: Elsevier; 2007.

4. Brasil. Ministério da Saúde. Secretaria de Vigilância Sanitária. Diretrizes de proteção radiológica em radiodiagnóstico médico e odontológico: regulamento técnico do Ministério da Saúde. Brasília: Ministério da Saúde; 1998.

5. Kreich EM, Queiroz MGS, Sloniak MC. Controle de qualidade em radiografias periapicais obtidas no curso de odontologia da UEPG. UEPG Biol Health Sci 2002 8(1):33-45.

6. Carvalho PL, Neves ACC, Medeiros JMF, Zõllner NA, Rosa LCL, Almeida ETDC. Erros técnicos nas radiografias intrabucais realizadas por alunos de graduação. RGO (Porto Alegre) 2009 abr-jun;57(2):151-5.
7. Gasparini AL, Lemke F, Carvalho AS, Cunha FL, Junqueira JLC, Tavano O. Verificação das condições do processamento radiográfico em consultórios odontológicos. 20052005 53(3):2179.

8. Matheus RA, Montebelo Filho A, Tanaka $E E$, Barros RM. Avaliaçäo no desempenho de acadêmicos durante tomadas radiográficas periapicais, pela técnica da bissetriz, realizada na Faculdade de Odontologia da Universidade Norte do Paraná (Unopar). RFO UPF 2000 jul-dez;5(2):25-9.

9. Fernandes AMM, Aguiar APS, Cruz LP, Aivazoglou MU, Gomes APM, Silva EG, et al. Avalilação dos erros radiográficos cometidos por alunos de graduação durante o tratamento endodôntico Rev odontol Univ Cid São Paulo (Online) 2010 set-dez ;22(3):216-22.

10. dos Anjos Pontual ML, Pinho Veloso $\mathrm{HH}$, dos Anjos Pontual A, da Fonseca Silveira MM. Errores en radiografias intrabucales realizadas en la Facultad de Odontología de Pernambuco-Brasil. Acta odontol venez 2005 enero;43(1):19-24.

11. Felippe MCS, Nassri MRG, Burgos PG, Freitas SFT, Lage-Marques JL. Qualidade de radiografias periapicais realizadas por Graduandos durante o tratamento endodôntico. RSBO 2009 6(1):63-9. 
12. Dias IM, Furiati PF, Santos EE, Barros CC, Devito KL. Análise de erros radiográficos cometidos por acadêmicos da Faculdade de Odontologia de Juiz de Fora. Arq odontol 2009 45(1):51-6.

13. Guimarães CS, Pontual AA, Khoury $\mathrm{HJ}$, Rodrigues CD, Estrela C, Silveira MMF. Qualidade de imagens radiográficas processadas em consultórios odontológicos e em laboratório $\mathrm{RO}$ BRAC 2011 abr;20(52):79-82.
14. Panella J. Fundamentos de odontologia-radiologia odontológica e imaginilogia. São Paulo: Guanabara Koogan; 2006.

15. Guimarães S, Costa C, Rosa R, Medici Filho E, Castilho J, Faig-Leite $\mathrm{H}$. Avaliação dos erros radiográficos mais comuns em uma clínica de radiodiagnóstico. Rev ABRO 2009 10(1):38-44.

Recebido 27/12/2013

Aceito 29/12/2013
MENDONÇA DM

AMORIM MEB

MEDINA PO

alVES FILHO AO

BRITO TCCA

CONDE NCO

AVALIAÇÃo DE ERROS EM EXA

MES RADIOGRÁFI-

COS INTRABUCAIS

REALIZADO POR

ACADEMICOS DE

ODONTOLOGIA / UFAM
REV, ODONTOL.

UnIV. CID, São

PAULO

2013; 25(3): 208 -

15，SET-DEZ 